\title{
Adverse Event Verbatim Description
}

National Cancer Institute

\section{Source}

National Cancer Institute. Adverse Event Verbatim Description. NCI Thesaurus. Code C78541.

The verbatim description of the adverse event. 